Conclusion Retrospective case-finding in General Pracice is feasible and the uptake for screening appeared very successful. Inclusion criteria were not strictly adhered to but identified persons with LTBI were offered non-funded treatment. Treatment completion rates were excellent. These Results will hopefully inspire on-going prospective screening of newly registered persons and prove to be more successful in the longterm than new entrant screening.

\begin{tabular}{|c|c|c|}
\hline IGRA results & Outcomes & $\begin{array}{l}\text { Number of } \\
\text { persons }\end{array}$ \\
\hline $\begin{array}{l}\text { Negative and borderline } \\
\text { negative }\end{array}$ & Discharged & 298 \\
\hline $\begin{array}{l}\text { Technical error/ } \\
\text { insufficient cells }\end{array}$ & Discharged & 7 \\
\hline Indeterminate & Referred to the local TB clinic & 5 \\
\hline \multirow{12}{*}{$\begin{array}{l}\text { Positive and borderline } \\
\text { positive }\end{array}$} & Referred to the local TB clinic & 50 \\
\hline & $\begin{array}{l}\text { Outcomes of the } 55 \text { referred to the } \\
\text { local TB clinic }\end{array}$ & \\
\hline & Diagnosis of active TB & 2 \\
\hline & Completed LTBI treatment & 33 \\
\hline & Stopped LTBI treatment & 2 \\
\hline & Declined LTBI treatment & 2 \\
\hline & Postponed treatment - pregnancy & 2 \\
\hline & Postponed treatment - other & 2 \\
\hline & $\begin{array}{l}\text { Not suitable for treatment } \\
\text { (previous active/latent treatment or due to } \\
\text { age) }\end{array}$ & 7 \\
\hline & Negative IGRA - 2nd test & 1 \\
\hline & DNA & 2 \\
\hline & Lost to follow up & 2 \\
\hline
\end{tabular}

\section{P166 DIAGNOSING PULMONARY TUBERCULOSIS: HOW USEFUL IS THE CHEST X-RAY REPORT?}

${ }^{1} \mathrm{KJ}$ Myall, ${ }^{2} \mathrm{~W}$ Owen, ${ }^{2} \mathrm{RA}$ Breen, ${ }^{1} \mathrm{~F}$ Perrin. ' $K$ King's College Hospital, London, UK; ${ }^{2}$ Guy's and St Thomas' NHS Foundation Trust, London, UK

\subsection{6/thoraxjnl-2017-210983.308}

Introduction and Objectives The diagnosis of pulmonary tuberculosis (TB) is frequently delayed, resulting in increased morbidity and mortality. One factor may be a delay in referral for chest X-ray (CXR). The NICE quality statement of January $2017^{1}$ suggests that all patients with imaging suggestive of active pulmonary tuberculosis are assessed by the next working day. We wanted to understand how many patients with confirmed TB have an initial CXR which correctly reported the diagnosis.

Methods We performed a retrospective analysis of the patient databases of two London hospitals of patients with confirmed pulmonary TB. We examined the report of the CXR prior to diagnosis in each case for mention of tuberculosis or referral to respiratory medicine.

Results 247 adult patients were treated for pulmonary TB between January 2014 and May 2017. Of these, 231 (94\%) had a CXR reported at our hospitals prior to diagnosis. 206 (89\%) patients had a CXR which was reported as abnormal and 106 (52\%) had a report which suggested TB as the diagnosis. In $29(12 \%)$, the report did not mention TB, but did suggest referral to a respiratory physician, meaning that overall, $65 \%$ of cases would have been assessed. 198 patients (86\%) had culture-positive disease, and of these, 91 (46\%) had a CXR which suggested the diagnosis. In 123 cases (50\%), the sputum was smear-positive for AFB and of these, 63 (51\%) had a CXR reported as TB. Of those patients whose CXR was reported as TB, $92(87 \%)$ had a positive culture, and 63 (51\%) were smear-positive.

Conclusions In our study, the initial CXR was abnormal in most cases of pulmonary tuberculosis, but in only $52 \%$ of these was TB suggested as the diagnosis. Thus almost half of diagnoses were missed on initial CXR, including those with smear-positive disease, and an automatic referral for assessment would not have been triggered. We think that these data highlight the importance of considering radiologist training as part of TB control efforts.

\section{REFERENCE}

1 National Institute for Health and Care Excellence. Tuberculosis 2017;QS141. Available at: nice.org.uk/guidance/qs141

\section{P167 THE USEFULNESS OF TABLET COUNTING TO IDENTIFY POTENTIAL TB TREATMENT NON-COMPLIANCE}

H Patel, Y Abunga, SO Brij. Peterborough City Hospital, Peterborough, UK

\subsection{6/thoraxjnl-2017-210983.309}

Background Early identification of non-compliance in patients taking anti-tuberculous therapy (ATT) may improve treatment outcomes and prevent the emergence of drug therapy resistance. Since 2012, the TB Pharmacy Team has been tablet counting to identify missed doses. Strategies to improve ATT compliance such as provision of dosette boxes, more frequent appointments and directly observed therapy (DOT) can then be initiated.

Aim To evaluate the usefulness of tablet counting as an effective strategy to help identify and reduce non-compliance with ATT.

Methods A retrospective review of active TB cases diagnosed between January 2012 to December 2016 was undertaken. Objective compliance was graded according to accuracy of tablet counting. Treatment outcomes were assessed clinically and subsequent change from normal practice identified.

Results 248 persons received at least 2 months ATT from the TB Clinic and were further evaluated. The majority of persons $(87.5 \%)$ were graded as fully $(61.3 \%)$ compliant (accurate tablet counting) or mostly (26.2\%) compliant (few missed doses) both with good clinical response. The commonest strategy to improve treatment outcome in $25(10.1 \%)$ with partial compliance (missed doses with adequate clinical response) was to increase duration of therapy. Poor compliance (missed doses, poor clinical response, more than 1 non-attendance) in 4 patients resulted in admission and completion of DOT as an in-patient. NICE guidance would have identified 22 persons for DOT. 19/22 received DOT (2 MDR-TB; 12 prisoners; 3 homelessness, 2 intravenous iv drug users). 3 (2 with mental health disorders; 1 alcohol dependence) successfully completed therapy with increased frequency of appointments and family involvement. Tablet counting identified 25 partially compliant persons (12 had no obvious risk factor; 5 prisoners on DOT; 2 persons with mental health disorders and 1 with alcohol dependence as above; 3 HIV; 2 pregnant). Treatment 
outcomes were adequate although $11 / 25$ were lost to followup (did not attend their end of treatment consultation) including 5 prisoners (released or transferred).

Conclusions Treating TB in prisoners and homeless persons continues to be a challenge, even when DOT is undertaken. Tablet counting can help identify potential non-compliance in persons without obvious risk factors. In our experience, tablet counting is an effective and relatively cheap objective adjunct in the assessment of ATT compliance.

\section{P168 SHOULD WE CONTINUE SCREENING HOUSEHOLD CONTACTS OF ALL INDEX CASES WITH TB IRRESPECTIVE OF INFECTIVITY?- AN ANALYSIS OF CONTACT SCREENING YIELDS STRATIFIED ACCORDING TO INDEX SITE OF DISEASE AND SMEAR STATUS}

R Enuechie, M Kanu, A Amoah, J Marshall, M Ogundengbe, F Ogunrin, LV Baker. University Hospital Lewisham, London, UK

\subsection{6/thoraxjnl-2017-210983.310}

Aim NICE Guidance (2016) recommends that TB contact screening is only carried out on close contacts (household and workplace/school contacts) of patients with infectious tuberculosis ie pulmonary tuberculosis (AFB smear positive) and laryngeal tuberculosis. However previous guidance recommended screening all household contacts of any index with TB irrespective of infectious status. The aim of this study was to look at the yields of contact screening amongst 3 groups of index cases- infectious smear positive pulmonary TB, smear negative pulmonary $\mathrm{TB}$ and extrapulmonary TB.

Method We analysed our records for contact screening of index cases with tuberculosis notified between January 2011 and May 2016. Index cases were divided into pulmonary smear positive, pulmonary smear negative and extrapulmonary. Contacts were divided into close, casual and workplace. The screening yields for each population were compared.

Results Between 1 st January 2011 and 31 st May 20161887 contacts of 408 notified index cases with TB were screened; 1109 were screened as contacts of smear positive pulmonary $\mathrm{TB}, 176$ contacts of smear negative pulmonary TB, 506 contacts of extrapulmonary $\mathrm{TB}$, the remainder the index site of disease was not specified. CXR screening was performed on the 510 contacts over the age of 35 (2011 guidelines). Patients 35 and under had 2 step immunological assessment with Mantoux and IGRA. There was a strong correlation between size of Mantoux response and IGRA positivity; 6\% of Mantoux $<6 \mathrm{~mm}, 23 \%$ Mantoux 6-10 mm, 40\% Mantoux 11-15 mm, 55\% Mantoux 15-20 mm, $84 \%$ Mantoux $\geq 25 \mathrm{~mm}$. 604 contacts of index cases with AFB smear positive sputum were assessed immunologically - 123 (20.3\%) were positive, 136 contacts of AFB smear negative pulmonary TB were assessed - $19(10.5 \%)$ were positive, and 383 contacts of extrapulmonary TB were assessed - 42 (11\%) were positive. 26 of 239 (11\%) workplace/school contacts of infectious TB were positive, compared to $21.5 \%$ of close/casual contacts.

Conclusions Although contact screening yields for index cases with smear positive pulmonary $\mathrm{TB}$ are high, the Results for extrapulmonary and smear negative pulmonary $\mathrm{TB}$ are not insubstantial. Our data would suggest that we should continue screening close contacts of all TB index cases irrespective of infectious status.

\section{P169 \\ LATENT TUBERCULOSIS INFECTION SCREENING OF ADULT CLOSE CONTACTS IN LONDON: A COST-UTILITY ANALYSIS}

${ }^{1} \mathrm{M}$ Hayama, ${ }^{1} \mathrm{~N}$ Green, ${ }^{2} \mathrm{SL}$ Seneviratne, ${ }^{3} \mathrm{M}$ O'Donoghue, ${ }^{4} \mathrm{~N}$ Drey, ${ }^{3} \mathrm{OM}$ Kon. ${ }^{1} \mathrm{NIHR}$ Health Protection Research Unit in Modelling Methodology and MRC Centre for Outbreak Analysis and Modelling, Imperial College London School of Public Health, London, UK; ${ }^{2}$ Clinical Immunology, Royal Free Hospital, London, UK; ${ }^{3}$ Chest and Allergy Department, St. Mary's Hospital, Imperial College NHS Trust, London, UK; ${ }^{4}$ School of Health Sciences, City, University of London, London, UK

\subsection{6/thoraxjnl-2017-210983.311}

Background The National Institute for Health and Care Excellence (NICE) guidelines in 2016 recommend tuberculin skin test (TST) at a $5 \mathrm{~mm}$ induration size cut-off for latent tuberculosis infection (LTBI) screening of adult close contacts of active tuberculosis (TB) cases. An alternative would be to use an interferon-gamma release assay (IGRA) which has a higher specificity, such as the QuantiFERON-TB Gold in Tube (QFTGIT) or T-SPOT.TB (T-SPOT). We aimed to evaluate the costeffectiveness of the screening and treatment of LTBI in adult close contacts with various combinations of these tests in a representative London cohort.

Methods Clinical data of adult close contacts of pulmonary TB cases who were recommended to receive TST and IGRA in a TB clinic in London between 2008 and 2010 were retrospectively reviewed. A Markov decision analytic model, using an NHS perspective and lifetime horizon, was used to compare costs and quality-adjusted life-years (QALYs) associated with 7 screening strategies followed by chemoprophylaxis: TST alone, IGRA (QFT-GIT or T-SPOT) alone, TST positive followed by IGRA, and TST negative followed by IGRA. Future costs and QALYs were discounted at 3.5\% per year.

Results 381 asymptomatic close contacts aged 18 to 65 years were included in this study. The mean age was 35.2 years and the majority $(75.3 \%)$ were BCG vaccinated. In the base-case analysis, QFT-GIT was the most cost-effective strategy with $£ 6876$ per QALY gained, compared to TST positive followed by QFT-GIT strategy. QFT-GIT alone averted 1.6 TB cases per 1000 contacts compared to TST positive followed by QFTGIT.

Conclusion Of the considered testing strategies, the QFT-GIT alone is preferable for LTBI screening in adult close contacts of pulmonary $\mathrm{TB}$ cases in London.

\section{P170 NEW ENTRANT LATENT TUBERCULOSIS SCREENING IN THE UK: SHOULD THE SEARCH BE WIDENED?}

MS Rana, S Sandhu, M Silka, SM Menzies. Wexham Park Hospital, Slough, UK

\subsection{6/thoraxjnl-2017-210983.312}

Introduction Most Tuberculosis (TB) cases in England are the result of reactivation of latent TB infection (LTBI) in foreignborn immigrants. The Collaborative TB Strategy recommends LTBI screening in New Entrants, who have entered the UK in the previous 5 years, are aged 16-35 and from countries with a TB incidence of $\geq 150$ cases/100,000 population. ${ }^{1}$ This is seen as cost-effective, ${ }^{2}$ but in contrast to National Institute for Health and Care Excellence (NICE) guidance, which recommends screening those aged 0-65 from countries with a lower TB incidence of $\geq 40$ cases/100,000). This study evaluated how the two screening programmes compared. 